\title{
A NOVEL CAPACITIVE SENSING PRINCIPLE FOR MICRODEVICES
}

\author{
Jian Zhou \\ Ronald N. Miles* \\ Shahrzad Towfighian \\ State University of New York at Binghamton \\ Binghamton, NY, 13905 \\ Email: jzhou41@binghamton.edu \\ miles@binghamton.edu \\ stowfigh@binghamton.edu
}

\begin{abstract}
Conventional capacitive sensing places significant limitations on the sensor design due to the pull-in instability caused by the electrostatic force. The main purpose of this study is to examine a low-cost novel capacitive sensing principle based on electrostatic balance which promises to avoid these design limitations. The approach uses an asymmetric electric field on a structure with fingers that can generate a repulsive force while the gap is low and create an attractive force while the gap is large. The size and thickness of the fingers are also responsible for creating repulsive or attractive forces on the structure. This approach has recently been applied successfully in the design of capacitive actuators to provide a repulsive driving force. A new design principle for capacitive sensing is described that avoids pull-in instability by designing the fingers such that the structure is at the equilibrium.
\end{abstract}

\section{INTRODUCTION}

Electrostatic transduction relying on simple capacitors is the most common sensing and actuation method in MEMS because of its simplicity and high efficiency [1-4]. Electrostatic MEMS include micro inertial sensors, pressure sensors, flow sensors, micro mirrors, switches, micro motors, etc. The most common forms of electrostatic sensing and actuation are based on either simple parallel-plate capacitors or comb-drive configurations. Due to the electrostatic force, the movable electrodes will overcome the restoring force of the supporting structure leading

\footnotetext{
* Address all correspondence to this author.
}

to the collapse to the fix electrodes if the applied voltage is too big or the gap between the aligned electrodes is too small. Conventional capacitive sensing and actuation thus places significant limitations on the sensor and actuator design due to the pull-in instability caused by the electrostatic force. Taking the capacitive sensing in miniature microphone for example, limitations lie in the following aspects [5]: 1) the electronic noise performance of the microphone cannot be improved freely by increasing the bias voltage due to the collapse of the diaphragm against the back plate; 2 ) in order to survive from the pull-in instability of the diaphragm, the sensitivity of the microphone is limited due to the small bias voltage and the large stiffness of the support; 3) the narrow air gap between the diaphragm and the back plate is a primary source of thermal noise.

Pull-in instability continues to become increasingly important for the design of electrostatic MEMS and NEMS devices $[1,6]$. Many experimental and theoretical studies have been done on the pull-in instability problem involved in actuation. And various approaches such as the pre-stress combdrive method [7], voltage control method [8], additional suppression comb electrodes [9], and offset-drive method [10] etc. have been presented to control the pull-in or extend the actuator travel range beyond the limitation of pull-in. Taking advantage of the electrostatic repulsive force, He and Ben Mrad proposed an electrostatic actuation principle which can enlarge the driving range without the limitation of pull-in. This principle has been applied successfully in the design of capacitive actuators [11-13].

A novel capacitive sensing principle is described in this paper inspired by the actuation method based on the repulsive force. As the actuation approach uses an asymmetric electric 
field on a structure with fingers that can generate a repulsive force while the gap is low and create an attractive force while the gap is large, this novel capacitive sensing principle can balance itself so as to overcome pull-in instability by designing the sensing structure such that it is at the equilibrium. A new concept for condenser microphone design is presented based on this novel approach.

\section{ILLUSTRATION OF THE REPULSIVE STRUCTURE}

As has been shown previously, groups of identical structures which are comprised of fixed fingers $f_{1}, f_{2}$, and movable fingers $f_{3}$ can provide a force on the movable fingers $f_{3}$ while the electrical potential of various fingers is different [13]. The structure which can generate a repulsive force is shown as Figure 1 . Suppose the number of movable fingers is $N$, the length of the finger is $L$, the thickness and width of the fingers are $t$ and $w$ respectively, the distance between the unaligned and fixed finger $f_{2}$ is $d$, the gap between $f_{2}$ and $f_{3}$ is $g$, the electrostatic force $F$ can be expressed as:

$$
F=\frac{N V^{2} L}{2} \frac{d C}{d g}
$$

Where $C$ is the capacitance of an elementary cell which is divided by Neumann boundary.

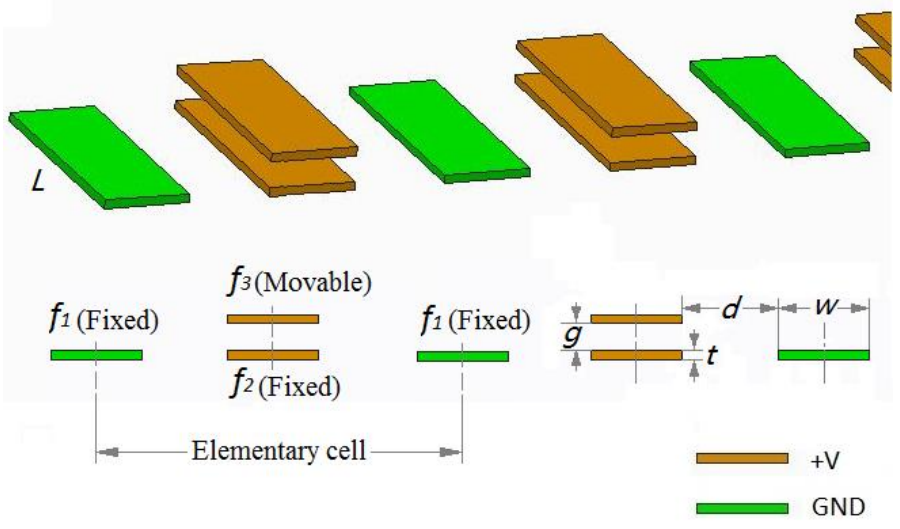

FIGURE 1. Repulsive structure

In the present study, the 2-D electric field is simulated numerically using a finite element approach (using COMSOL). In the simulation, the thickness $t$, width $w$, distance $d$ and length $L$ of all the fingers are $0.5 \mu m, 5 \mu m, 5 \mu m, 1 m$ respectively. The electric potential of the single cell divided by Neumann boundary is considered to be the same as there are many group of elementary cells. Surfaces of the $f_{1}$ are grounded while the electric potential on the surfaces of $f_{2}$ and $f_{3}$ is $1 V$. A larger boundary with zero surface charge density is established in the outside of the electrodes. The material of the electrodes is silicon, while the rest of the material in the boundary is air.

The electric field of the repulsive structure is shown in Figure 2(a) while the gap $g$ is $2 \mu \mathrm{m}$. Figure 2 (b) shows the surface electric field of the movable finger $f_{3}$. The electrical field is asymmetric on the upper and bottom surface of the movable finger $f_{3}$ which will generate a repulsive force. (a) Single elementary cell

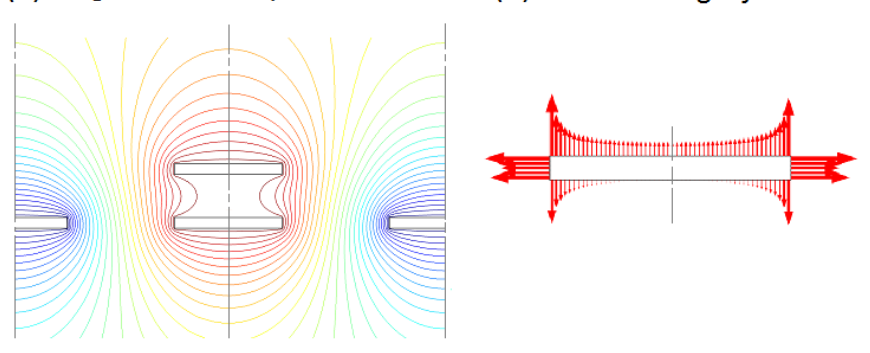

Figure 2. Electric field

\section{SENSING PROPERTIES CHARACTERIZATION}

The properties characterization including the electrostatic force, the capacitances of various parts, and the surface charge density are studied in the following to illustrate the principle of the novel capacitive sensing.

The electrostatic force $f_{3}$ per unit length of the movable finger described above is shown as Figure 3. This simulation result agrees well with those of the previous of the repulsive capacitive actuator [12]. The repulsive force decreases as the gap $g$ increases from zero and turns into an attractive force if the gap $g$ exceeds about $5.5 \mu \mathrm{m}$.

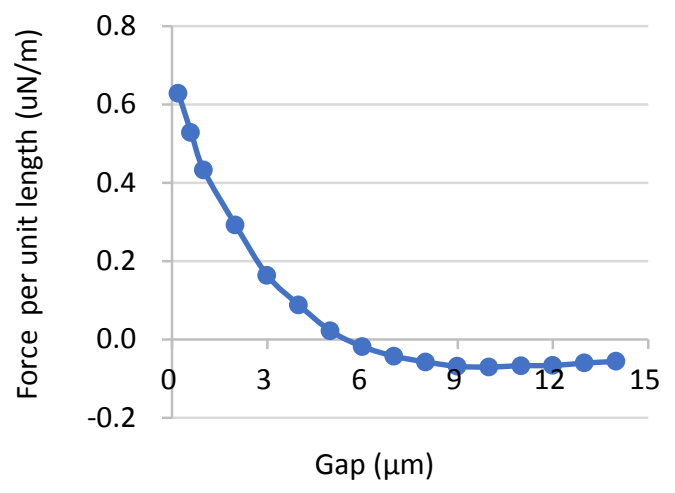

FIGURE 3. Force variation with gap

The capacitance of an elementary cell can be expressed by the following equation:

$$
C=\frac{\delta A}{V}
$$

Where, $A$ is the surface area, $V$ is the applied voltage, and $\delta$ is the surface charge density, which can be obtained by simulation.

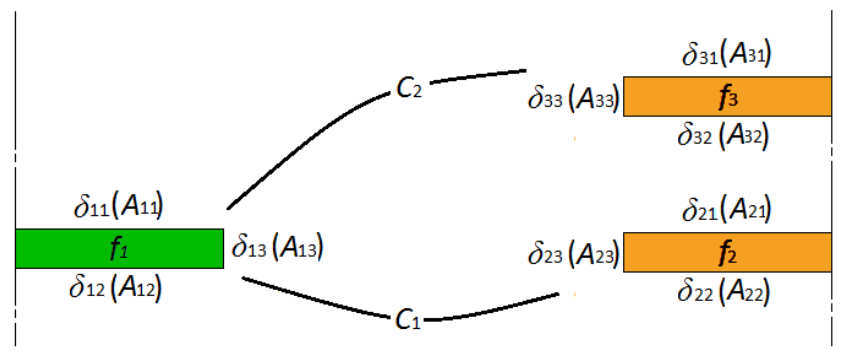

FIGURE 4. Capacitance and surface charger density 
The composition of the capacitance and surface charge density in the half of an elementary cell is shown in Figure 4. For the half of an elementary cell, its capacitance is composed of the capacitance $C_{1}$ (Capacitance between $f_{1}$ and $f_{2}$ ) and $C_{2}$ (Capacitance between $f_{1}$ and $f_{3}$ ). As the total capacitance $C$ of an elementary cell is twice of the half one, so it can be expressed as the following equation:

$$
C=2 C_{1}+2 C_{2}
$$

Capacitance variations as a function of the gap $g$ in an elementary cell are shown in Figure 5. Because the length of the structure is much longer than the width, the capacitance at the end surface of the structure is ignored. Capacitance between $f_{1}$ and $f_{3}$ decreases with the gap while capacitance between $f_{1}$ and $f_{2}$ increases, and their combined action contributes the increment of the total capacitance $C$ while the gap is small. However, the total capacitance $C$ drops with increasing gap while the gap distance is larger than $5.5 \mu \mathrm{m}$.

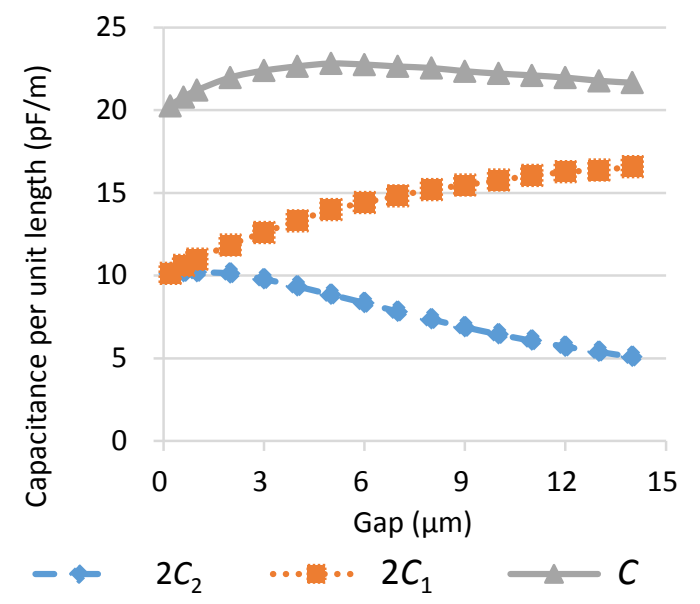

FIGURE 5. Capacitance variation with gap in an elementary cell

As the surface charge density of various surface areas on various fingers can be obtained by simulation, we can calculate the capacitances between various fingers. Capacitance in the equation (3) can be expressed as:

$$
\begin{aligned}
& C=2\left(\delta_{11} A_{11}+\delta_{12} A_{12}+\delta_{13} A_{13}\right) / V=2 \delta_{1} A_{1 / 2} / V \\
& C_{1}=\left(\delta_{21} A_{21}+\delta_{22} A_{22}+\delta_{23} A_{23}\right) / V=\delta_{2} A_{1 / 2} / V \\
& C_{2}=\left(\delta_{31} A_{31}+\delta_{32} A_{32}+\delta_{33} A_{33}\right) / V=\delta_{3} A_{1 / 2} / V
\end{aligned}
$$

Where $A_{1 / 2}$ is the total area of a half finger and $\delta_{1}, \delta_{2}, \delta_{3}$ are the effective surface charge density value of various fingers. According to (3)-(6), the surface charge density value of $f l$ can be expressed as:

$$
\delta_{1}=\delta_{2}+\delta_{3}
$$

The sensing principle we investigate is based on using asymmetric electric field on the moving finger $f_{3}$, which creates repulsive force for small gaps and generates attractive force for large gaps, as shown in Figure 3. Hence, it can be designed to balance itself to avoid pull-in.
As the capacitance of the sensor is determined by the geometry of the structure, the sensing structure design will decide the performance of the capacitive sensor. To move a step further for the sensor design, we have studied the surface charge density $\delta$ of the various finger surfaces with an applied the unit voltage, $1 \mathrm{~V}$, to investigate the influence of the structure geometry. The surface charge density variation with gap $g$ is shown in Figure 6, where Figure 6(a) is the effective surface charge density $\delta_{1}, \delta_{2}, \delta_{3}$ of various fingers as shown in figure 4 . Being a property of electric field shown as equation (7), the surface charge density $\delta_{1}$ is the sum of $\delta_{2}$ and $\delta_{3}$.

Figure 6(b) shows the surface charge density $\delta_{32}$ and $\delta_{21}$ of the aligned surfaces between $f_{1}$ and $f_{2}$. When the gap is very small, the difference between surface charge density of the two areas is approximately zero. As the gap increases, the surface charge density of the two surface areas deviates rapidly which means that charges flow to the stationary electrode rather than the moving electrode. Because of the difference in charge concentration on the two surfaces as the gap increases, the repulsive force changes to the attractive force as shown in Figure 3.

Figure 6(c) shows the surface charge density of the four upper and bottom surfaces which are unaligned. The bottom of $f_{1}$ and $f_{2}$ have similar surface charge density, $\delta_{22}$ and $\delta_{12}$ almost keep unchanged with the variation of the gap. The surface charge density $\delta_{31}$ in the upper of $f_{3}$ is sensitive to gap change and decreases rapidly. The surface charge density $\delta_{11}$ of $f_{1}$ increases while the gap is small and decreases while the gap is large.

Figure 6(d) demonstrates the surface charge density $\delta_{13}, \delta_{23}$, $\delta_{33}$ on the sides of the fingers. The surface charge density $\delta_{13}, \delta_{23}$, $\delta_{33}$ on the side areas are larger than that of other surface areas talked above when the gap is small.

From the surface charge distribution, we can explain the change in the nature of the force seen in Figure 3. As the gap increases, the surface charge density $\delta_{32}$ of the bottom surface of $f_{3}$ increases at first and then decreases as shown in 6(b). At the same time, the surface charge density $\delta_{31}$ and $\delta_{33}$ in the upper and side surface of $f_{3}$ are always decreasing shown as $6(c)$. The surface charge density change with the variation of the gap $g$ contributes to the electrostatic force change shown in figure 3 .

\section{ESTABLISHMENT OF THE SENSOR MODEL}

As mentioned above, the sensing structure can always be balanced at the equilibrium by the combined effect of the electrostatic force and the elastic force of the supporting beams. Figure 7 shows the sensor model with the capacitive sensing structure. In this figure, (a) shows the in-operation state of the sensor, (b) is the state when the sensor is electrified, and (c) depicts the detection of the signal such as the sound pressure $p(t)$.

Parameters in this figure are interpreted as the following:

$k$ : Stiffness of the structure;

$g_{0}$ : Initial gap distance;

$x_{0}$ : The distance of the equilibrium position from the initial position after electrification;

$F_{\mathrm{e} 0}$ : Electrostatic force after electrification; 
(a) Effective Surface charge density of each finger

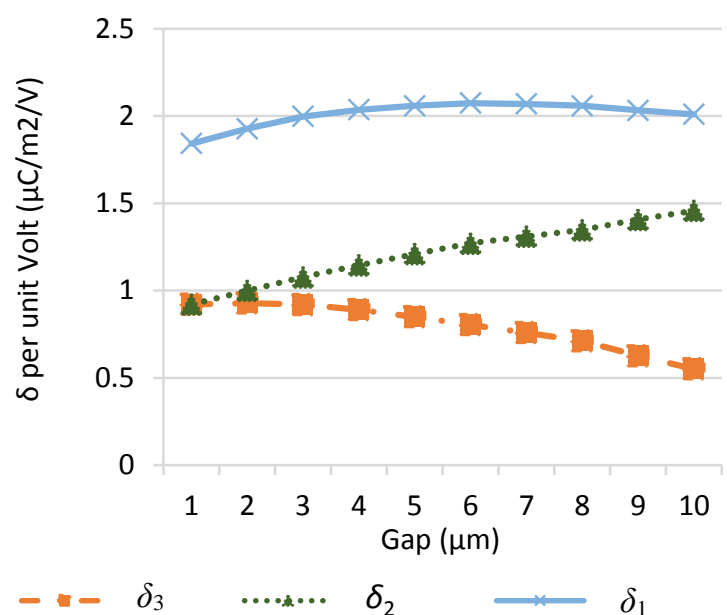

(c) Surface charge density of unaligned areas

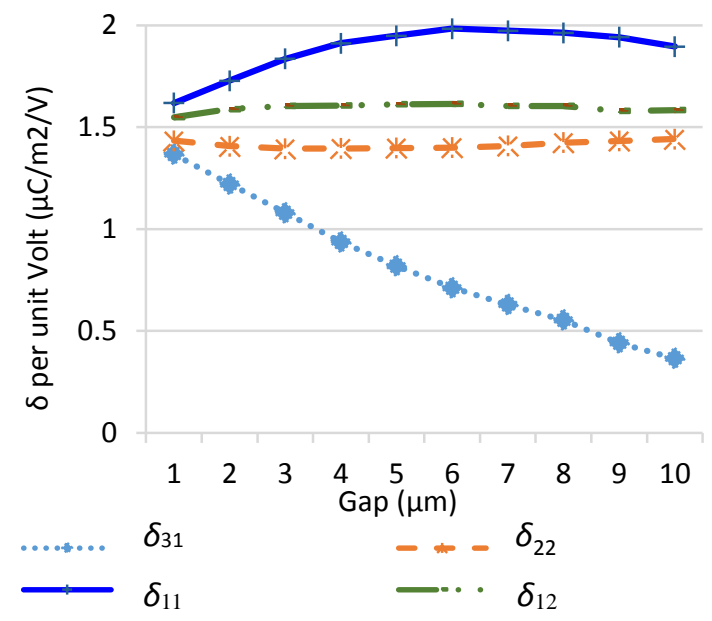

(b) Surface charge density of aligned areas

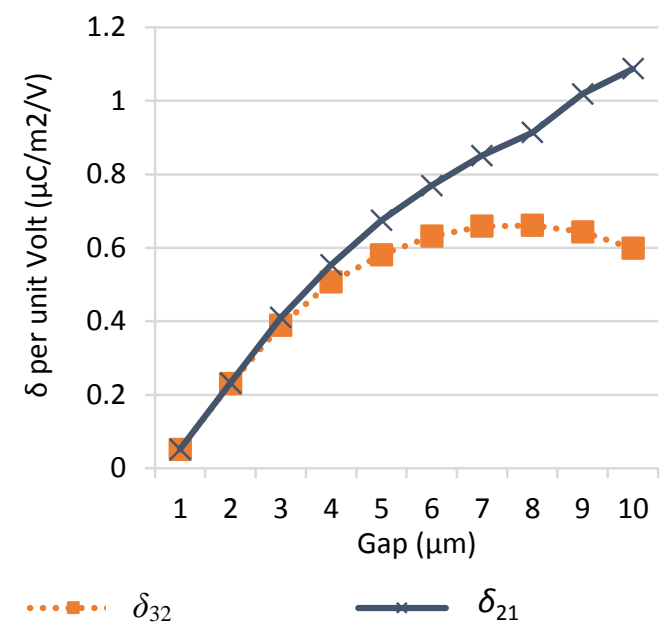

(d) Surface charge density of side areas

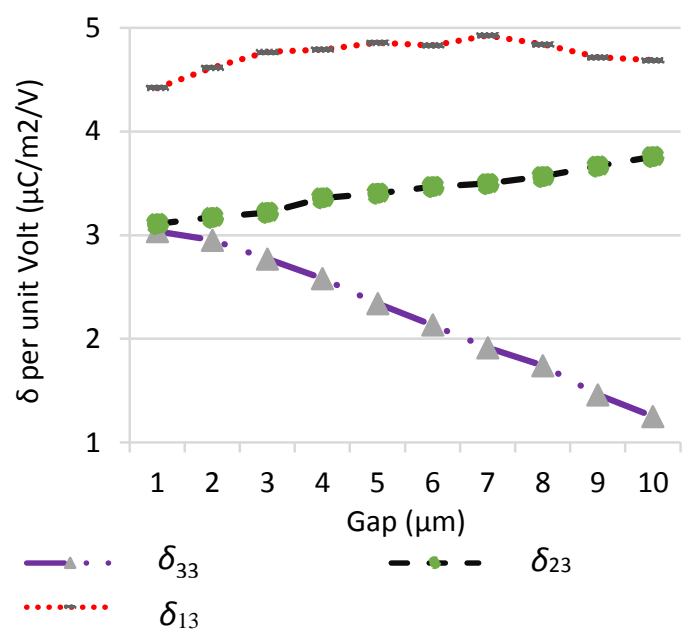

FIGURE 6. Surface charge density variation with gap

$F$ : Sensing signal such as the force caused by the sound wave pressure $p(t)$;

$\Delta F_{\mathrm{e}}$ : Variation of electrostatic force to the sound wave. After electrification, the prestress force $F_{o}$ of the diaphragm caused by the electrostatic force can be written as:

$$
F_{0}=k x_{0}=F_{e 0}
$$

Under the influence of the sound wave pressure $p(t)$, the deformation $x$ of the diaphragm is:

$$
x=\frac{F-\Delta F_{e}}{k}
$$

In order to increase $x$, the stiffness $k$ will be designed to be very small. Due to the electrostatic force, the capacitive sensing structure will be moved to the equilibrium after electrification automatically. At this working status, the sensor will avoid pullin instability. To design $x_{0}$ to be small, the prestress force $F_{\mathrm{o}}$ should be small, which suggest the initial gap should be chosen at the distance where the electrostatic force $F_{\mathrm{e} 0}$ is 0 .

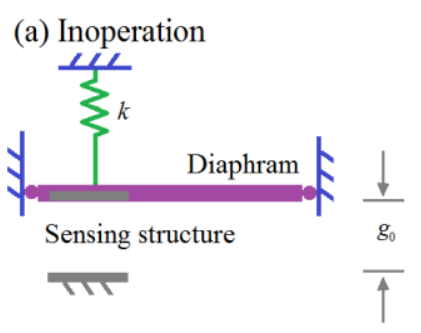

(b) Electrification

(c) Detection of the sound wave

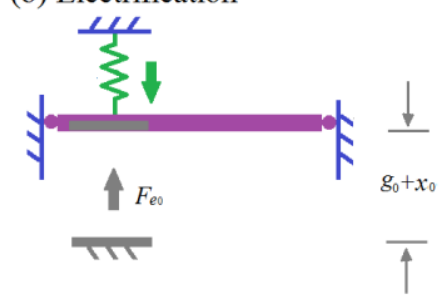

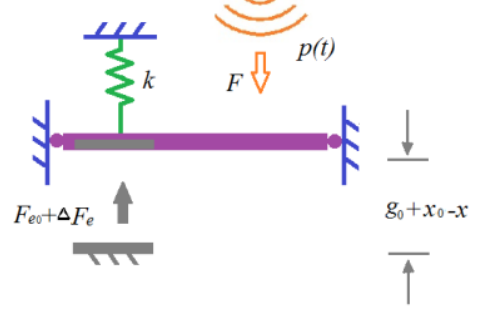

FIGURE 7. Sensor model 
There are various parameters which will influence the initial balance gap distance of the electrostatic force and the performance of the capacitive sensing, such as the applied voltage $V_{1}\left(f_{1}\right.$ and $\left.f_{2}\right), V_{2}\left(f_{1}\right.$ and $\left.f_{3}\right)$, and the inherent structure parameters including the size of the elementary cell, the thickness $t$, and the distance $d$. To apply the present sensing approach more effectively, in the following we examine the effects of the elementary cell size on the capacitance and electrostatic force. The effects of enlarging the elementary cell size of the previous simulation whose width is $5 \mu \mathrm{m}$ by a factor of 2 on the force and capacitance are shown as Figure 8 and 9, respectively. As the width of a single finger examined previously is $5 \mu \mathrm{m}$, the enlarged finger width is $10 \mathrm{um}$. The change rate of the force and capacitance to the gap variation will decrease when the cell size is bigger.

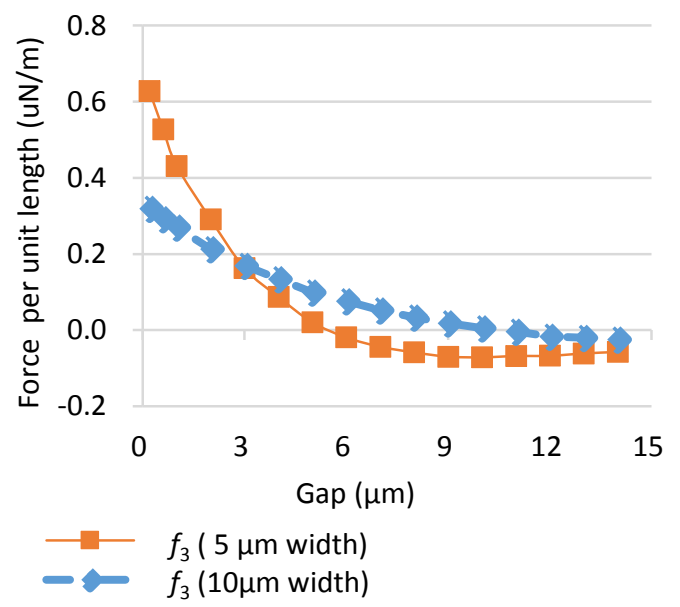

FIGURE 8. Force comparison of various width size

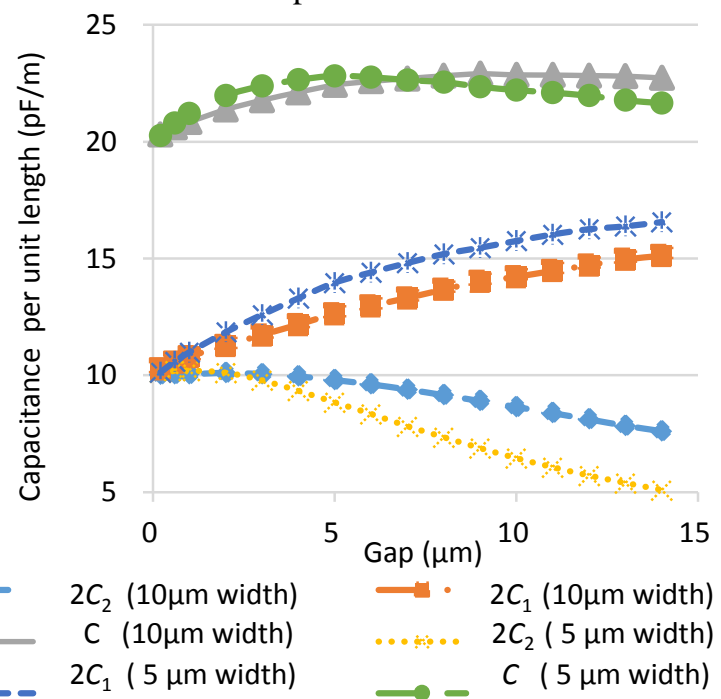

FIGURE 9. Capacitance comparison of various size in an elementary cell

In addition, the balance gap position of the electrostatic force is increased with the increase of the cell size. Shown as
Figure 8 , the balance gap position is about $11 \mu \mathrm{m}$, which is 2 times larger than the balance gap $5.5 \mu \mathrm{m}$, when the size scale is enlarged by 2 times. So it is better to choose the small elementary cell size in order to make the sensor more sensitive. Moreover, we can design more groups of elementary cells in a specific total sensor size if each elementary cell size is small.

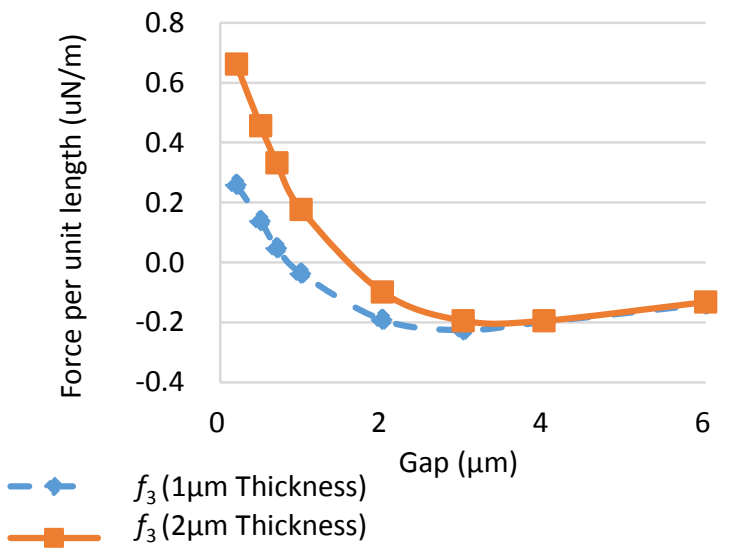

FIGURE 10. Force comparison of various thickness

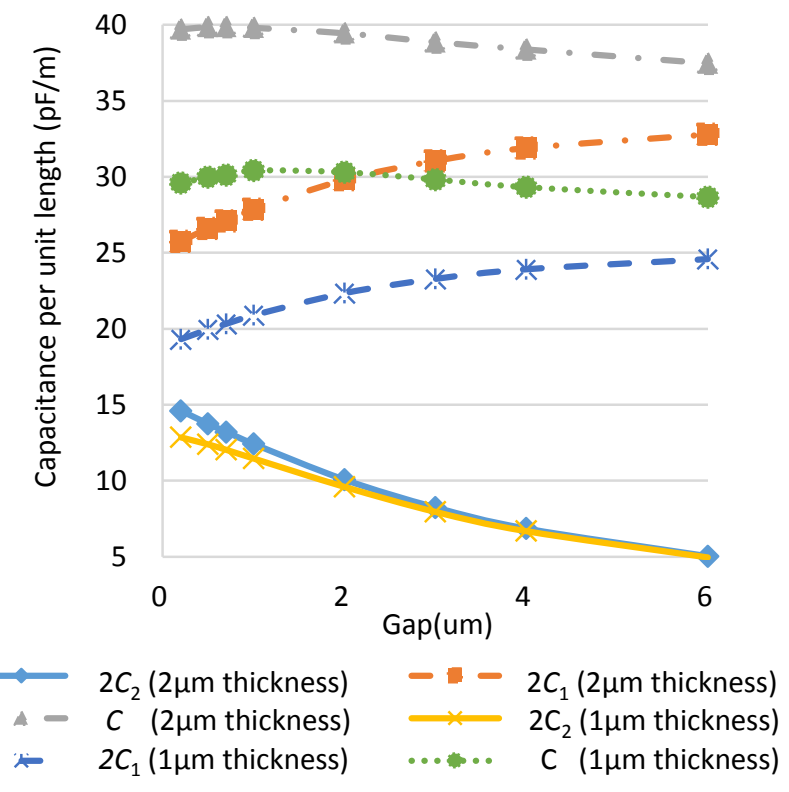

FIGURE 11. Capacitance comparison of various size in an elementary cell

As the surface charge density of the side surfaces are high, the influence of the size surfaces on the force and capacitance is dramatic if the size of thickness and the width are similar. Figures 10 and 11 show the force and capacitance comparison when the thickness of the sensing structures are different. The width $w$, distance $d$ of the finger are both $2 \mu \mathrm{m}$, and the thickness are $1 \mu \mathrm{m}$ and $2 \mu \mathrm{m}$ in the simulation. As shown in figure 10 , the force balance position is larger when the thickness is $2 \mu \mathrm{m}$. During sensor design, the initial balance position of the electrostatic force can be modified by changing the thickness of the fingers. 


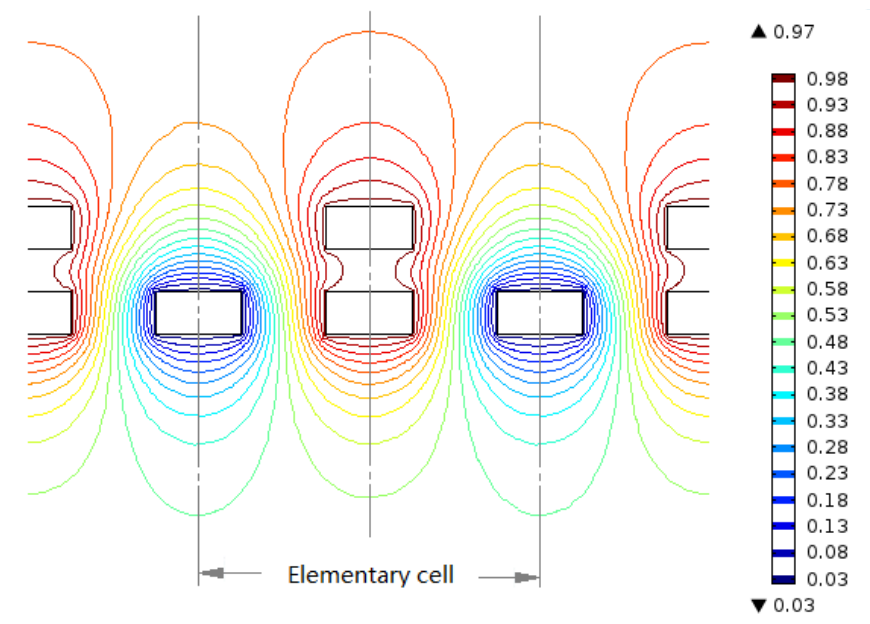

FIGURE 12. Electric potential of the sensing fingers

All the capacitances of various fingers in an elementary cell are larger when the thickness is $2 \mu \mathrm{m}$, as shown in figure 11 . The total capacitance and the capacitances of the F2 are increased uniformly as the increment of the thickness, while the movable finger F3 capacitance stays approximately constant. Figure 12

\section{REFERENCES}

[1] Younis MI., 2011, MEMS Linear and Nonlinear Statics and Dynamics, Springer, New York, NY, Chap. 3.

[2] Hak MG., 2006, MEMS Applications, CRC Press, Boca Raton, FL, Chap.2.

[3] Bleckmann H., et al., 2014, Flow sensing in air and water, Springer, Berlin, GER, Chap. 17.

[4] Bi HC., 2013, "Ultrahigh Humidity Sensitivity of Graphene Oxide," Sci. Rep., 3, (12p).

[5] Miles RN, Hoy RR., 2006, “The Development of a Biologically-inspried Directional Microphone for Hearing Aids," Audiol Neurootol., 11(2), pp. 86-94.

[6] Zhang WM., et al., 2014, "Electrostatic Pull-in Instability in MEMS/NEMS: A Review," Sens. Actuators A, 214, pp. 187-218.

[7] Chiou JC., Lin YJ., 2005, “A Novel Large Displacement Electrostatic Actuator: Pre-stress Comb-drive Actuator," J. Micromech. Microeng., 15(9), pp. 1641-1648. shows the electric potential of the sensing fingers while the thickness is $1 \mu \mathrm{m}$.

\section{CONCLUSION}

A novel capacitive sensing principle based on electrostatic balance is described in this paper. The concept is based on repulsive force fingers introduced before by $\mathrm{He}$ at al [11-13]. The simulation results based on finite element analysis using COMSOL indicates that this capacitive sensing approach can avoid pull-in instability. Instead of conventional parallel-plate electrodes, elementary sensor cells are used that consist of aligned, unaligned and moving fingers. The elementary cell of the sensor is investigated from the area charge density perspective to analyze the force and capacitance on the fingers. The different distributions of the surface charge density in the various surface areas of the movable finger contribute to the generation and variation of the electrostatic force which can be used to keep the sensing structure at equilibrium. The performance of the sensing structure and the balance position of the electrostatic force may be adjusted by reducing the size scale of the elementary cell and modifying the thickness of the fingers. Further work should be done to apply this novel capacitive sensing approach into practice.

[8] Towfighian S., et al., 2011, “A Large-stroke Electrostatic Micro-actuator,” J. Micromech. Microeng., 21(7), (12pp).

[9] Hou., et al., 2006, "Extending Displacements of Comb Drive Actuators by Adding Secondary Comb Electrodes," J. Micromech. Microeng., 16(4), pp. 684-691.

[10] Su J., et al., "A Surface Micromachined Offset-drive Method to Extend the Electrostatic Travel Range," J. Micromech. Microeng., 20(1), (10pp).

[11] He SY., Ben Mrad R., Chong J., 2011, "Repulsive-force Out-of-plane Large Stroke Translation Micro Electrostatic Actuator," J. Micromech. Microeng., 21(7), (12p).

[12] He SY., 2006, "Novel Micro Electrostatic Actuators with Applications," Ph.D. thesis, University of Toronto, Ontario, Canada

[13] He SY., Ben Mrad R., 2008, “Design, Modeling, and Demonstration of a MEMS Repulsive-Force Out-of-Plane Electrostatic Micro Actuator," J. Microelectromech. S., 17(3), pp.532-547. 